
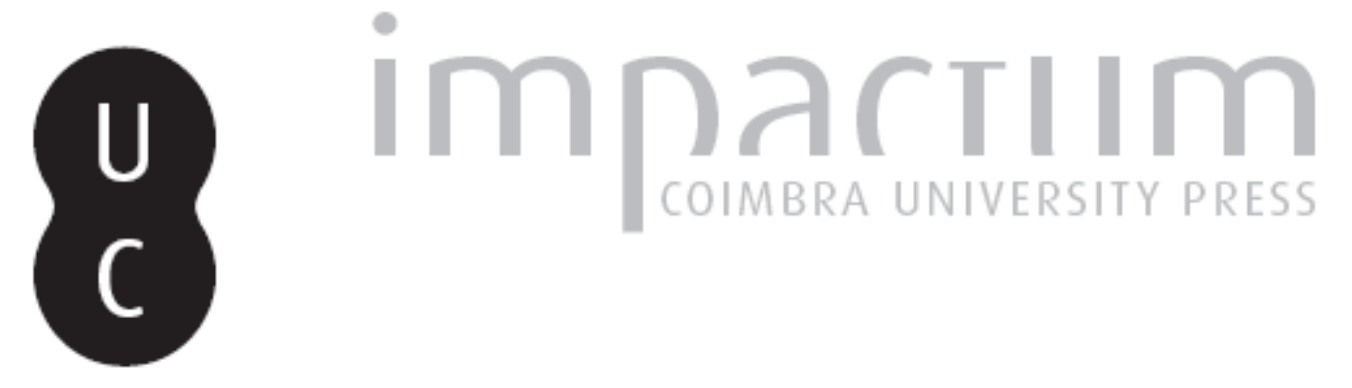

\title{
«Revetir la vie des chiens», l'animal comme modele moral
}

\section{Autor(es): Husson, Suzanne}

Publicado por: Annablume Clássica

URL persistente:

URl:http://hdl.handle.net/10316.2/24518

DOI:

DOI:http://dx.doi.org/10.14195/1984-249X_11_6

Accessed : $\quad$ 26-Apr-2023 11:01:57

A navegação consulta e descarregamento dos títulos inseridos nas Bibliotecas Digitais UC Digitalis, UC Pombalina e UC Impactum, pressupõem a aceitação plena e sem reservas dos Termos e Condições de Uso destas Bibliotecas Digitais, disponíveis em https://digitalis.uc.pt/pt-pt/termos.

Conforme exposto nos referidos Termos e Condições de Uso, o descarregamento de títulos de acesso restrito requer uma licença válida de autorização devendo o utilizador aceder ao(s) documento(s) a partir de um endereço de IP da instituição detentora da supramencionada licença.

Ao utilizador é apenas permitido o descarregamento para uso pessoal, pelo que o emprego do(s) título(s) descarregado(s) para outro fim, designadamente comercial, carece de autorização do respetivo autor ou editor da obra.

Na medida em que todas as obras da UC Digitalis se encontram protegidas pelo Código do Direito de Autor e Direitos Conexos e demais legislação aplicável, toda a cópia, parcial ou total, deste documento, nos casos em que é legalmente admitida, deverá conter ou fazer-se acompanhar por este aviso.

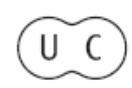



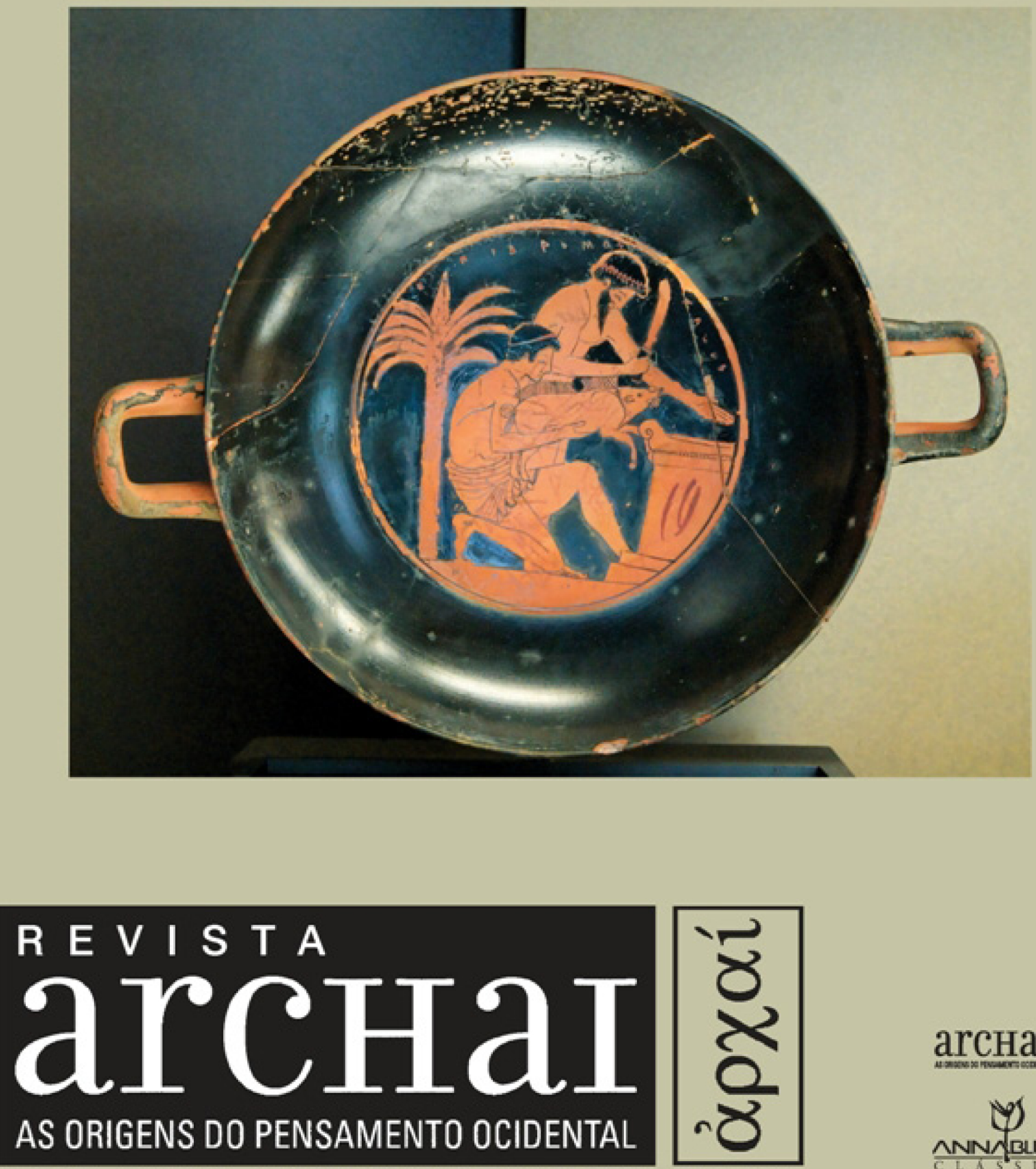

ARCHAI JOURNAL: ON THE ORIGINS OF WESTERN THOUGHT

arcHaI凰

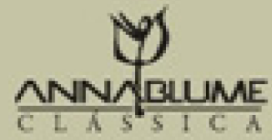




\section{«REVETIR LA VIE DES CHIENS», L'ANIMAL COMME MODELE MORAL}

HUSSON, S. (2013). "Revetir la vie des chiens", l'animal comme modele moral. Archai, n. 11, jul-dez, p. 69-78.

RÉSUMÉ: Si la référence des cyniques anciens à l'animal comme modèle est bien connue, il est plus difficile d'en comprendre le sens, car ils ne sont pas les seuls dans l'Antiquité à évoquer l'animal dans un contexte éthique. En partant de la définition aristotélicienne du paradigme, il est dans un premier temps montré que l'animal intervient parfois chez les cyniques dans le cadre d'une induction qui présuppose qu'homme et animal appartiennent à un même genre moral. Mais l'animal apparaît également, dans d'autres contextes, à titre d'exemplum épidictique : il montre de façon immédiate et évidente à l'homme la vertu et le genre de vie qu'il doit imiter.

Ces deux types d'exemplarité reposent sur une conception analogique des rapports entre animalité et humanité, typique de la pensée antique, mais l'originalité du cynisme, par rapport, par exemple, au platonisme, réside dans le fait que la vertu attribuée à l'animal n'est pas conçue comme intrinsèquement inférieure à celle de l'homme. Cependant, comme toute imitation, l'imitation de l'animal repose sur une interprétation préalable de ce qui en lui est à imiter. Ce cercle herméneutique explique que, parfois, l'animal dans le cynisme ait un statut de paradigme négatif.

MOTS-CLÉS: animal, cynisme, paradigme, modèle, exemplarité.

ABSTRACT: The ancient Cynics reference to animals as moral models is a well known fact, nevertheless it is difficult to understand its meaning, since the Cynics were not the only ones who mentioned animals in an ethical context. The first
* Université Paris-Sorbonne Paris IV, Centre Léon Robin

$$
\text { Paris }
$$

1. Dans ce chapitre l'épicurien résume les Républiques de Diogène de Sinope, fondateur du cynisme et de Zénon de Citium, fondateur du stoïcisme, qui fut élève de Cratès le Cynique, lui-même disciple de Diogène. Les premiers stoïciens reprirent, dans une certaine mesure, la référence au modèle animal, mais en l'intégrant à leur contexte doctrinal (cf. GOULET-CAZÉ, 2003).
Suzanne Husson*

Diogène le Cynique, dans sa République, si nous en croyons Philodème, recommandait de « revê-

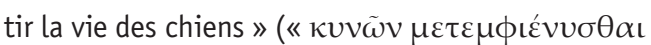
ßíov »; PHILODÈME, Sur les stoïciens, 7, XVIII, 5-7 $)^{1}$. Plus largement, la référence au modèle animal est une constante de la pensée cynique. Or, que l'animal puisse être un modèle pour l'homme est une idée qui semble à la fois familière et étrange. Du récit homérique de l'attachement du chien d'Ulysse (Od. XVII 290 sq.), jusqu'à nos jours, on n'a cessé, par exemple, d'admirer la fidélité du chien, sa constance plus parfaite que celle de l'homme. Néanmoins il semble difficile au philosophe contemporain de penser que le chien, ou en général l'animal, puisse être considéré comme un modèle à imiter.

Des obstacles théoriques considérables, en effet, s'y opposent, le premier d'entre eux résidant dans l'hétérogénéité des statuts éthiques entre l'animal et l'homme. Si nous pouvons, en effet, considérer que nous partageons la sensibilité avec les animaux qui en sont capables, nous seuls sommes capables de poser consciemment des normes qui ne soient pas un simple prolongement ou une reproduction du donné, mais qui le transcendent de façon explicite. Ainsi, l'attachement du chien, certes, est touchant et cet affect témoigne d'une forme de communauté sensible nous unissant, néan- 
part of this paper deals with the Aristotelian definition of the paradigm as a rhetorical version of the induction. It can apply to some occurrences of the animal model in cynic literature, but in this case it is presupposed that humans and animals belong to the same moral kind. Moreover, animal can be found, in other contexts, as an epidictic exemplum, showing us virtue and the way of life we have to imitate, in a direct and obvious way.

Those two types of exemplarity rely on an analogical conception of the relationship between bestiality and humanity, typical of ancient thought. But Cynicism is original, compared, for example, to Platonism, because the virtue, ascribed to animals by the Cynics, is not intrinsically inferior to human virtue. Nevertheless, as every imitation, the imitation of animals relies on a preliminary interpretation of what we are supposed to imitate. This hermeneutic circle explains the fact that, sometimes, animals in Cynicism appear as negative paradigms.

KEYWORDS: animal, cynicism, paradigm, model, exemplarity.

moins l'animal ne peut devenir un modèle dans le sens où il réaliserait de façon plus parfaite ce que nous sommes appelés à accomplir. La permanence de son attachement est inscrite dans des schémas de comportement hérités, alors que la fidélité humaine est le produit d'une décision autonome et à tout moment révocable, et c'est ce qui, malgré son inconstance, en fait justement la valeur. Un même comportement est conçu comme émanant de consciences radicalement distinctes, de telle sorte que parler de comportement vertueux chez l'animal reviendrait à céder à notre tendance à attribuer, de façon anthropomorphique, nos états subjectifs à des réalités qui en sont incapables.

D'autre part, même si, nous situant aux franges du monde animal qui nous sont le plus apparentées et dans la volonté d'abolir les frontières qui nous en séparent, nous reconnaissions à certaines espèces le statut de sujets éthiques, nous serions bien en peine de savoir où trouver des modèles de comportement tant ceux-ci différent, ne serait-ce qu'au sein des sociétés de primates ${ }^{2}$. Ainsi, l'animal ne peut apparaître comme un modèle pour l'homme qu'à un titre purement métaphorique, l'homme projetant sur lui des normes et des idéaux proprement humains : ce n'est pas l'animal qui nous montre ce que nous devons être, mais nous qui parfois le construisons à notre image, ou plutôt à l'image de notre représentation de nous-mêmes.

Cependant, pour Diogène, « revêtir la vie des chiens » n'était pas une simple métaphore, puisque cela impliquait précisément de transgresser bon nombre des limites symboliques séparant l'animal de l'humain, comme l'abolition des tabous sexuels et en particulier celui de l'inceste, la justification de la nudité, de l'anthropophagie ou bien encore le rejet des rites mortuaires ${ }^{3}$. Dans quel sens, donc, l'animal et, en particulier, le chien, pouvait-il être constitué en paradigme éthique par les cyniques ? Nous nous appuierons, dans un premier temps, sur la définition aristotélicienne du paradigme.

\section{L'animal et le paradigme inductif}

Pour le Stagirite, en effet, l'exemple n'est que la version rhétorique de l'induction ${ }^{4}$, celle-ci impliquant la capacité d'établir des rapports de ressemblance au sein d'un genre :

Nous avons dit que l'exemple est une induction

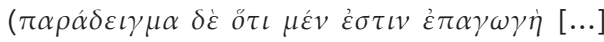
$\varepsilon \iota ّ \rho \tau \alpha \iota)$ [...] L'exemple ne présente les relations ni de la partie au tout, ni du tout à la partie, ni du tout au tout, mais seulement de la partie à la partie, du semblable au semblable, lorsque les deux termes rentrent dans le même genre, mais que l'un est plus connu que l'autre; par exemple: Denys aspire à la tyrannie, puisqu'il demande une garde ; autrefois, en effet, Pisistrate, ayant ce dessein, en demandait une, et, quand il l'eut obtenue, il devint tyran; de même Théagène à Mégare; et tous les autres que l'on connait deviennent des exemples pour Denys, dont pourtant on ne sait pas encore si c'est pour cette raison qu'il demande une garde. Tous ces cas particuliers rentrent sous la même notion générale que l'aspirant à la tyran-

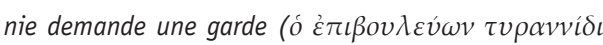

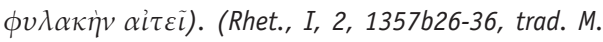
Dufour [très légèrement modifiée])

L'exposé d'un exemple implique donc l'évocation d'au moins deux cas semblables (ici
2. Les structures socio-sexuelles vont en effet de la monogamie (gibbons) aux sociétés multimâles et multifemelles (macaques), en passant par les sociétés en harems (babouins) ou, au contraire, polyandres (tamarins) ; cf. COPPENS ; PICQ, 2001, chap. 5 « Vivre en groupe »; par VAN H0OF, J.A.R.A.M., en particulier le tableau p. 205. Quant au système humain il n'est « ni franchement polyandre, ni franchement polygame » (ibid., p. 241). Dans la conclusion de cet ouvrage (ibid., p. 518, col. 2) P. Picq, ironise sur le fait qu'aujourd'hui les bonobos, avec leur activité sexuelle débridée, jouent le rôle du « bon sauvage » du XVIIIe, alors que les chimpanzés dont les mœurs sont plus violentes, exemplifient les travers de l'humanité. Quoi qu'il en soit, il convient de ne pas céder à l'erreur naturaliste car, contrairement à l'animal, l'humanité choisit son destin : " ce que nous sommes ou croyons être est notre affaire, et les grands singes n'ont rien à y voir » (ibid., p. 519, col. 1).

3. Pour l'analyse de ces aspects de la République de Diogène, cf. HUSSON, 2011, part. II, chap. 2. 4. De la même façon que l'enthymème est la traduction rhétorique du syllogisme, cf. Aristote, Rhet., I, 2, 1356b1-16, An. post., I, 1, 71a1-11. 
Pisistrate et Théagène) dans un même genre, entre lesquels on peut établir un rapport d'analogie qui va être appliqué au cas à connaître, lui-même implicitement considéré comme faisant partie du même genre. Comme chez Pisistrate, la demande d'une garde personnelle était liée chez Théagène à la volonté de devenir tyran, on peut donc en tirer la loi générale suivante : " quiconque demande une garde aspire à la tyrannie », exprimée par la formule ambiguë « l'aspirant à la tyrannie demande une garde » qui ne signifie pas que « tout aspirant

5. Cf. trad. M. Dufour ad. loc. Le fait de se pourvoir d'une armée personnelle était déjà considéré par Platon comme une étape nécessaire de l'accession à la tyrannie dans un contexte démocratique (Rsp., VIII, 556b). 6. Voir aussi Aristote, Rhet., II, 20, 1393a31-b4.

7. Dion Chrysostome, ibid., 29.

8. Á cette induction implicite s'ajoute un raisonnement a fortiori, puisque non seulement les animaux évoqués ont la peau plus fragile que celle de l'homme, mais ils sont de plus capables de vivre dans un milieu encore plus hostile : l'eau froide.

à la tyrannie demande une garde $»^{5}$, mais qu'en général, c'est l'aspirant à la tyrannie qui demande une garde. Si l'on s'exprimait dans un cadre scientifique ou dialectique il faudrait compléter pas le syllogisme suivant : « or Denys demande une garde, donc il aspire à la tyrannie »; mais comme nous sommes dans un contexte rhétorique, l'énoncé des deux exemples, assorti de l'application de l'analogie à l'exemple à connaître, suffit ${ }^{6}$.

Or, nous pouvons trouver une utilisation analogue de l'exemple animal dans la littérature cynique. Ainsi, dans le discours VI (6) (25-28) de Dion Chrysostome nous trouvons ce propos attribué à Diogène :

Voici pourquoi, à ce qu'il lui semblait, le mythe raconte comment Zeus châtia Prométhée à la suite de la découverte et du don du feu : parce qu'il constituait le commencement et l'origine de la mollesse et de la sensualité des hommes. En effet, Zeus assurément ne hait pas les hommes et ne veut pas les priver d'un bien (26). Lorsque certains disaient qu'il n'est pas possible que l'homme vive comme les autres animaux, à cause de la fragilité de sa chair et parce qu'il est nu, recouvert ni de poils comme un grand nombre de bêtes, ni de plumes, ni enveloppé par une peau épaisse, à ces arguments, il répliquait que (27) c'est à cause de son genre de vie que l'homme est si fragile, en effet, il fuit la plupart du temps le soleil, ainsi que le froid. D'autre part, la nudité de son corps ne le gênait nullement et

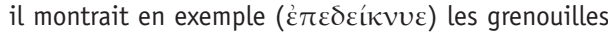
et de nombreux autres animaux beaucoup plus fragiles que l'homme, beaucoup plus nus, dont quelques uns supportent non seulement l'air hivernal, mais aussi peuvent vivre dans l'eau la plus froide. (28) Il montrait

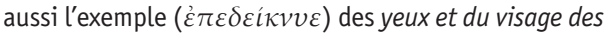
hommes eux-mêmes qui n'ont pas besoin de protection et généralement aucun animal ne naît dans un lieu où il ne puisse vivre.

Cette contestation du mythe de Prométhée et d'Épiméthée utilise à deux reprises l'exemple pour réfuter l'idée d'une différence fondamentale entre la condition humaine et la condition animale, qui justifierait le recours de l'humanité à la technique. Épiméthée n'a pas oublié l'homme dans sa répartition des dons, de sorte que le vol prométhéen du feu n'était pas le bienfait qu'imagine l'opinion commune, bien (avant deuxième citation) au contraire.

En courant après ce qui est agréable, ils [c-à-d. les hommes] mènent une vie toujours plus désagréable et plus pénible et alors qu'ils semblent tout prévoir pour eux-mêmes (prometheisthai), ils se corrompent de la pire façon par cet intense souci et prévoyance (prometheia). ${ }^{8}$

La première série d'exemples est destinée à montrer que l'absence de poils ou de plumes ne rend pas nécessaire le recours au vêtement ou aux techniques de construction susceptibles de nous abriter des intempéries. Le texte signale que les cas évoqués sont nombreux, ce qui montre bien qui nous sommes dans un contexte inductif. De même que s'agissant des grenouilles et d'autres animaux le fait d'avoir la peau fragile ne les empêche pas de supporter le froid, ceci est aussi vrai de l'homme ${ }^{8}$. Les exemples sont ensuite choisis dans le corps humain lui-même : de même que les yeux et le visage n'ont pas besoin de protection contre le froid, il en va de même pour les autres parties du corps puisque, comme le disait Aristote, nous progressons, dans l'exemple, de partie à partie. Ces séries d'exemples sont censées aboutir à la proposition générale : « Aucun animal ne naît dans un lieu où il ne puisse vivre ».

Ainsi l'exemple animal est utilisé pour établir le fait qu'animal et homme font partie d'un même genre, non seulement au niveau biologique (ce sont des animaux), mais également au niveau éthique : ils sont, de façon semblable, appelés à mener un genre de vie naturel. 
Une autre utilisation d'exemples d'animaux est prêtée par Dion à Diogène :

[CEdipe] comprit qu'il s'était uni à sa mère et qu'il avait eu des enfants d'elle et, par la suite, il fallait sans doute le cacher ou bien légaliser la situation auprès des Thébains, mais dans un premier temps il révéla tout, puis s'en scandalisa, en hurlant de toutes ses forces qu'il était à la fois le père et le frère de ses enfants, le mari et le fils de la même femme. Or les coqs ne se scandalisent pas de ces choses, ni les chiens ni aucun âne, ni les Perses qui passent pour être le meilleur peuple d'Asie. (Discours $X$ : Diogène ou Des domestiques 29-30).

La réaction d'CEdipe à la révélation de son inceste n'était pas pertinente car, d'une part, ni les coqs, ni les chiens, ni les ânes n'évitent l'inceste et pourtant ils n'en souffrent pas, il n'est donc pas un mal pour eux. Il en va de même, parmi les hommes, des Perses, donc un homme comme CEdipe n'avait pas à éviter l'inceste, ou à en éprouver de la culpabilité. Les exemples d'animaux ${ }^{9}$ suggèrent par induction que nul animal ne souffre de l'inceste. Le cas des Per$\operatorname{ses}^{10}$ permet d'étendre l'induction au genre humain et de répondre à l'objection de ceux qui réserveraient l'interdit de l'inceste à l'humanité. L'exemple animal est ainsi utilisé pour montrer que l'animal et l'homme doivent se soumettre aux mêmes règles de vie. Cette figure rhétorique repose sur le présupposé implicite que tous deux appartiennent au même genre moral, qu'ils sont d'une condition similaire, poursuivant un bien de même ordre. En tant que tel ce présupposé ne fait l'objet d'aucune démonstration, mais c'est le propre même de toute induction : celle-ci présente la vérité d'une proposition universelle par l'examen de cas particuliers qui sont censés manifester leur évidence par eux-mêmes. Le champ des cas susceptibles de produire l'induction ne peut donc pas être questionné pour lui-même, puisque l'induction procure la connaissance des principes premiers qui rendent toute démonstration possible ${ }^{11}$. L'effet du discours cynique n'est pas tant de démontrer que de déplacer le champ de l'évidence, de rapprocher ce que l'opinion éloigne, en élargissant le domaine de l'induction morale.
Cependant l'exemple n'intervient pas seulement à titre de substitut de l'induction. Le modèle moral, en effet, n'a pas besoin d'une énumération de cas similaires pour manifester son exemplarité. Sa seule excellence, immédiatement manifeste, appelle l'imitation.

\section{L’animal, paradigme épidictique}

Avec Cicéron et Plutarque, en effet, l'exemple change de sens, il ne représente plus la partie émergeante d'un processus inductif, mais la mise en évidence d'une perfection morale éclatante, d'autant plus éclatante qu'elle est unique. Malgré son unicité, ou plutôt à cause d'elle, l'exemplum se propose à nous comme modèle. Comme le dit Plutarque :

La vertu [...] par les actes qu'elle inspire, nous dispose aussitôt, non seulement à admirer les belles actions, mais en même temps, à rivaliser avec ceux qui les ont accomplies [...] C'est que la beauté morale attire activement à elle et suscite aussitôt dans l'âme un élan vers l'action; elle ne forme point les mœurs de celui qui la contemple par la seule imitation, mais elle détermine nos résolutions par la connaissance pratique de la vie active. (PLUTARQUE, Vie de Périclès, 2, 2-4, 153b1-10, trad. R. Flacelière et é. Chambry)

Selon l'heureuse expression de G. Jeanmart, nous sommes là face au « pouvoir performatif de la vertu elle-même » ${ }^{12}$ qui par sa manifestation suscite le désir de l'acquérir, voire l'action immédiate, car, comme le disait Sénèque «Longue est la route par le précepte, courte et facile par l'exemple» ${ }^{12}$. L'exemple ainsi conçu n'a pas vocation à démontrer, ou plutôt à participer à la démonstration, comme dans le genre judiciaire, mais à montrer, comme dans le genre épidictique ${ }^{14}$.

Or, l'anecdote emblématique de Diogène apprenant la sagesse d'une souris relève de cet usage de l'exemple :

C'est parce qu'il avait, à en croire Théophraste dans son Mégarique, vu une souris qui courait de tous côtés, sans chercher de lieu de repos, sans avoir peur de l'obscurité ni rien désirer de ce qui passe pour
9. Nous pouvons également remarquer que Diogène recourt à l'exemple d'animaux domestiques, tout simplement parce que leurs comportements sont connus de tous.

10. Là aussi, nous somme face à un argument $a$ fortiori. Les Perses ne condamnent pas l'inceste, alors qu'ils sont censés être un peuple excellent.

11. Cf. Aristote, An. post., I, 18, 81a38-b9.

12. Art. cit., p. 14.

13. "Longum iter est per praecepta, breve et efficax per exempla » (Lettres à Lucilius, I, 6, 5).

14. Cf. la formule de Quintilien, III, IV, 13 : « Je crois que le terme 'épidictique' implique moins démonstration qu'ostentation »,

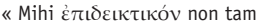
demonstrationis vim habere quam ostentationis videtur », trad. J. Cousin dans Quintilien, Institution oratoire, t. II, Livres II et III (1975), Paris, Les Belles Lettres. 
des sources de jouissance, que Diogène découvrit un remède aux difficultés dans lesquelles il se trouvait.

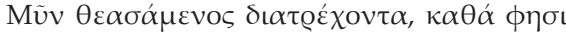

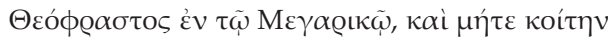

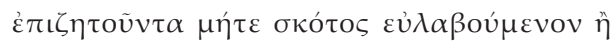

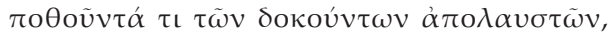

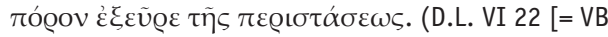
172], trad. M.-0. Goulet-Cazé) ${ }^{15}$

L'animal devient, non pas enseignant, comme il pouvait l'être chez Démocrite, pour qui l'homme inventa les techniques en imitant l'animal ${ }^{16}$, mais éducateur, puisque cette anecdote met en scène la conversion de Diogène à la philosophie. Les autres cyniques le deviennent par la fréquentation d'un maître, mais dans la tradition Diogène apprend le cynisme des personnages les plus inattendus, par exemple d'un enfant qui lui montre comment se passer de son écuelle (cf. SSR VB156-161), et plus généralement de cette souris. Avant même que la théorie rhétorique n'en soit faite, Théophraste lui fait jouer le rôle d'un exemplum, mais d'un exemplum silencieux manifestant par son seul comportement quel doit être l'état intérieur dans lequel le sage doit se trouver. Diogène en regardant simplement l'animal comprend quels sont ses désirs et ses craintes et ainsi ce qu'il ne faut ni craindre ni désirer, à savoir ce qui passe dans l'opinion pour une source de crainte ou de jouissance. L'efficacité de l'exemple est immédiate, puisqu'il est censé donner naissance à une nouvelle sagesse. Certes cela implique que, d'une part, la souris soit heureuse, d'autre part que le bonheur humain et le bonheur animal soient de même nature, deux présupposés que l'exemple ne peut par lui-même justifier. Mais ce sont là deux objections que l'on peut adresser à n'importe quel exemple moral posé en modèle, et non au seul exemple animal. Comment être sûr, en effet, que l'individu humain donné en exemple a effectivement réalisé l'idéal moral qu'il prétend représenter ? Comment, d'autre part, un individu, pris dans des circonstances singulières, pourrait-il imiter les actions, non moins singulières, d'un autre individu, entre deux singularités, toute analogie n'est-elle pas artificielle ${ }^{17}$ ? Ainsi, dans une culture qui admet la valeur morale et mimétique de l'exemplarité comme l'était la culture antique, prendre l'animal pour modèle ne présente pas de difficulté insurmontable, d'autant plus qu'on pensait en général le rapport entre l'homme et l'animal selon la figure de l'analogie. Mais comment cette analogie était-elle pensée dans le cynisme et quelles en sont les conséquences pour les statuts relatifs de l'animal et de l'homme?

\section{Paradigme et analogie}

Selon l'analyse de P. Descola (2005, p. 319337 , en particulier le tableau, p. 323), la culture occidentale contemporaine conçoit la relation entre l'homme et les autres êtres vivants à partir d'une « différence des intériorités » associée à une « continuité des physicalités», autrement dit la pensée animale et pensée humaine y sont vues comme radicalement distinctes, alors que les corps des uns et des autres, composés des mêmes éléments matériels, y ont le même statut ontologique. Mais ce rapport « naturaliste » à la nature n'a émergé qu'à l'époque moderne : dans l'Antiquité et jusqu'à la Renaissance, l'homme et la nature étaient conçus dans un cadre « analogiste ». Celui-ci implique une « différence des intériorités », pensée non pas dans le cadre d'une dichotomie sans nuance séparant la conscience humaine du reste des vivants, mais dans celui d'une discontinuité graduelle, une échelle des aptitudes de pensée de plus en plus complexes. Il en va de même des « physicalités » qui, dans un contexte analogiste, sont également conçues sur un mode scalaire. Et entre les degrés de l'échelle des intériorités et des physicalités, il est possible d'établir des relations analogiques.

Et, en effet, c'est bien sur la possibilité d'établir des analogies entre les différents niveaux et échelles du réel que Platon établit sa propre conception du paradigme. S'il « est difficile [...] d'éclaircir de façon suffisante quelque sujet d'importance sans faire usage de paradigmes » (Pol., 277d1-2), il faut pour cela qu'il puisse exister des correspondances, des rapports de proportion, entre les différents plans du réel ${ }^{18}$. Mais l'analogie à la base du paradigme est la plupart du temps telle que l'être pris comme paradigme est situé à un niveau ontologique ou moral inférieur, par rapport à celui où se situe la 
chose à connaître : le paradigme, en effet, doit faire partie des objets sensibles (Pol., 285e1), il est un sujet mineur et plus facile (Soph., 218d1) qui nous prépare à la compréhension des questions difficiles. Ainsi, à la fin du Politique, l'art du tisserand est pris comme paradigme pour penser l'art du politique, alors que le tissage n'est qu'un des multiples arts qui lui sont subordonnés ${ }^{19}$, il en va de même lorsque le soleil est pris comme image du Bien au livre VI de la République (506d8-509c2). Nous pouvons dire qu'alors l'analogie est ascendante, dans la mesure où le paradigme, d'un niveau inférieur, conduit à la connaissance d'un niveau supérieur.

Or, tel est le cas de l'animal lorsque Platon l'évoque comme paradigme moral et politique. Le chien apparaît, par exemple, à deux reprises dans la République : tout d'abord au livre II (375c6-376b9), lorsqu'il s'agit de sélectionner le naturel des gardiens qui devront manifester un caractère à la fois doux et agressif, naturel paradoxal, que nous pouvons arriver à penser, parce qu'il se rencontre aussi chez le chien. Il reparaît ensuite au livre V (451d1-9), au moment de déterminer si les femmes pourront être gardiennes :

Ainsi croyons-nous que les femelles des chiens de garde doivent veiller comme les mâles sur les troupeaux, chasser avec eux et faire tout en commun, ou qu'elles doivent garder le logis, incapables d'autre chose que d'enfanter et d'élever des petits? (Rsp., 451d.)

Mais, quelle que soit l'utilité du paradigme animal pour déterminer la norme de l'action humaine, il n'en reste pas moins que l'animal demeure inférieur à l'homme. En effet, alors que, comme l'homme, il possède une partie désirante et courageuse, l'exercice de la partie rationnelle de l'âme lui fait défaut, à tel point que dans les livres centraux de la République, qui lui sont spécifiquement consacrés, le paradigme animal est abandonné ${ }^{20}$. De ce fait, dans une telle analogie ascendante, l'animal peut fort bien jouer le rôle d'analogon éthique de l'homme, sans que leurs situations hiérarchiques en soient renversées.

Or, de telles analogies animales abondent dans l'Antiquité. Plutarque, par exemple rapporte que :
Encore un seul exemple (paradeigmati) [...]. Lycurgue, le législateur de Lacédémone, prit deux chiots de la même portée et les éleva de manière dissemblable : il rendit l'un gourmand et vorace, l'autre apte à suivre une piste et à chasser. Puis, un jour que les Lacédémoniens étaient rassemblés : «C'est un facteur déterminant pour atteindre la vertu, ô Lacédémoniens, dit-il, que les habitudes, la formation, les leçons reçues, les principes de conduite d'une vie, et je vais sur le champ vous le rendre parfaitement sensible (fanera). » Ayant alors fait venir les deux chiens, il les lâcha après avoir disposé à leur portée et devant eux un plat de nourriture et un lièvre. L'un s'élança sur le lièvre, l'autre bondit sur le plat. Comme les Lacédémoniens n'arrivaient pas encore saisir la signification qu'avait à ses yeux cette exhibition de chiens et à quoi elle tendait, « Ils ont tous deux les mêmes parents », dit-il, « mais une éducation différente a fait de l>un un gourmand, de lsautre un chasseur ». (PLUTARQUE, De l'éducation des enfants 4, M. 2F-3B, trad. J. Sirinelli) ${ }^{21}$

Nous ne sommes pas ici en présence d'un exemple épidictique, puisqu'il requiert une explication de la part de l'orateur ${ }^{22}$, mais dans un cas d'induction à partir d'un cas particulier, fondé sur une similitude prétendue entre le dressage animal et l'éducation des enfants, caractérisés tous deux par une grande plasticité des données naturelles de départ. Cependant, même si le mode de vie imposé à l'animal sert de paradigme à l'éducation humaine, il n'est nullement question d'égaliser leur statut. D'ailleurs si Plutarque entreprend de dépeindre les vertus des animaux constitués en exempla dans son traité Que les bêtes brutes usent de la raison où Gryllos, le lieutenant d'Ulysse, transformé en cochon, "seul animal du corpus plutarquien à parler en philosophe » (BRÉCHET, 2005 , p. 44) ${ }^{23}$, refuse que Circé lui rende sa forme humaine parce que notamment, l'animal est plus courageux, tempérant et prudent que l'homme, il n'en demeure pas moins que pour le philosophe, l'animal, malgré sa vertu reste inférieur à l'homme. Il faut concevoir plutôt une échelle continue des capacités rationnelles, commençant à l'animal et culminant chez le sage ${ }^{24}$, de sorte que c'est dans les Vies parallèles, dont les héros sont humains,
19. Il fait, en effet, partie des causes auxiliaires du politique : Pol., 288b4-8.

20. Cf. HUSSON, 2011, p. 49-51. 21. L'anecdote est aussi rapportée dans les Apophtegmes laconiens, M. 225F-226B.

22. Alors que celui de la souris parlait de lui-même.

23. L'argumentation prêtée à Gryllos est d'un caractère composite, puisqu'on y retrouve des éléments cyniques, épicuriens et, dans une moindre mesure, platoniciens (BRÉCHET, 2005, p. 50 sq.). L'opposition entre Ulysse (partisan de la suprématie de l'homme) et Gryllos (qualifié de sophiste en 989 F) est d'ordre littéraire. Plutarque ne partage aucune des thèses radicales de ses deux personnages : l'animal est bien capable d'intelligence et de vertu, sans toutefois égaler l'homme (ibid., p. 58). 24. Cf. GONTIER, 1999, p. 90 101. Ainsi, dans De l'amour de la progéniture, les vertus des animaux, se conformant à une nature simple et sans mélange (493c), ont pour fonction naturelle, non pas la sauvegarde des animaux eux-mêmes, mais la production d'un sentiment de honte chez les êtres humains qui voudront alors les imiter (494f). Néanmoins, l'exemple moral de l'animal a des limites car seul l'homme est capable de justice (495b). Billault (2005, p. 42) va dans le même sens. 
que l'homme pourra trouver les exemples de vertu les plus abondants.

Qu'en est-il alors dans le cynisme ? D'après Diogène Laërce, Diogène le Cynique « répétait à cor et à cri que la vie accordée aux hommes par les dieux est une vie facile, mais que cette facilité leur échappe, car ils recherchent gâteaux de miel, parfums et raffinements du même genre » (D.L. VI 44 (SSR VB322), trad. M.-0. Goulet-Cazé). à ce titre, l'animal montre donc directement à l'homme, qui spontanément s'en détourne, quelle est la vie facile qu'il lui appartient de mener. Il lui est donc moralement supérieur, car humains et animaux sont destinés à poursuivre un bien de même ordre : la vie facile accordée par les dieux. Cette hiérarchie peut être indirectement confirmée par la sentence cynique « le propre des dieux est de n'avoir besoin de rien, de celui qui est semblable aux dieux d'avoir de peu de choses » (D.L. VI 105 (SSR VA 135)), nous avons là un principe de classement clair dont le degré maximum est l'autarcie des dieux. Sur cette échelle, l'animal se situe au dessus de l'homme, puisqu'il réalise immédiatement le degré d'autarcie dont il est capable. Néanmoins, comme le remarque Thierry Gontier ${ }^{25}$, dans un autre contexte l'animal n'est pas en aussi bonne place.

Quand il regardait les pilotes, les médecins, et les philosophes, il pensait que l'homme était le plus avisé des animaux; en revanche s'il regardait les interprètes des songes, les devins et leur cour, et tous les gens infatués de gloire et de richesse, alors il ne savait rien de plus fou que l'homme. (DL VI 24 (SSR VB375)).

Or, de fait, cette sentence ne peut pas constituer un argument cynique en faveur de la supériorité de l'homme. Un cynique, en effet, condamne en général les techniques, de sorte que l'art de la médecine ou du pilotage sont des travaux inutiles, voire corrupteurs : si, comme nous l'avons vu, aucun animal ne naît dans un endroit où il ne puisse vivre, il est évident qu'il ne sert à rien de savoir piloter un bateau et il en va de même pour la médecine car, comme Dion le fait dire à Diogène, les animaux vivent aussi longtemps qu'il leur est naturel de vivre, et ceci en bonne santé, sans recourir au médecin ${ }^{26}$.
Si donc cette sentence doit être réellement attribuée à Diogène, ce que l'on peut raisonnablement mettre en doute, il nous faut plutôt la rapprocher d'autres propos que Dion lui prête :

Sinon comment les premiers hommes auraient-ils pu être sauvés : il n'y avait ni feu, ni maisons, ni vêtements, ni d'autre nourriture que celle qui apparaît spontanément? Mais l'habileté des hommes, le fait de découvrir et d'inventer beaucoup de choses pour améliorer l'existence, ne fut pas très utile aux générations suivantes. En effet, les hommes se sont servis de leur savoir (sophia), non pas en vue du courage ou de la justice mais en vue du plaisir. (ibid. 28-29).

L'habileté, la capacité à inventer des hommes n'est pas remise en cause. L'homme est le plus avisé de tous les animaux parce qu'il sait plus que les autres trouver les moyens pour poursuivre les fins qu'il se donne. Néanmoins, plus que les animaux, il est aussi capable de se tromper, comme les devins qui s'imaginent pouvoir prédire l'avenir et les ambitieux qui s'imaginent que la gloire et la richesse les rendra heureux. Sa supériorité technique ne le rend ainsi pas moralement supérieur. L'humanité, en général, est inférieure à l'animalité, car le bonheur de l'homme et de l'animal sont de même ordre : réaliser la vie facile que la nature rend possible ; or, alors que l'homme se détourne de lui, l'animal le réalise spontanément.

Néanmoins sans doute Thierry Gontier a-t-il raison en avançant que le sage est supérieur à l'animal, non pas tant à cause de ce passage ambigu que de la reprise, par le cynisme, de l'idée socratique $d^{\prime}$ une amitié entre les sages et les dieux. Diogène disait ainsi que :

Tout appartient aux dieux ;

les sages sont amis des dieux ;

tout est commun entre amis ;

donc tout appartient aux sages. (D.L. VI 37 (SSR= VB 353)) $)^{27}$

Or nous ne trouvons pas de trace littéraire d'une éventuelle amitié entre les dieux et les animaux. De la sorte, même s'il est difficile de 
comprendre en quel sens les cyniques se référaient ainsi aux dieux ${ }^{28}$, force est de constater que le sage est présenté comme l'être le plus proche d'eux. La hiérarchie ${ }^{29}$ est donc la suivante : après les dieux viennent les sages, c'est-à-dire les hommes véritables, ensuite viennent les animaux, et enfin les hommes ordinaires. De sorte que l'homme ordinaire qui chercherait un modèle moral autour de lui, s'il ne trouve pas de cynique, doit se tourner vers l'animal et non pas vers ses contemporains, ou de grands hommes du passé. Il n'est pas, en effet, appelé à poursuivre un modèle de perfection, l'amenant à développer pour elles-mêmes des capacités le distinguant de l'animal, mais à user de sa raison dans le seul but de dénoncer l'illusion au cœur des désirs humains ordinaires, afin de parvenir à la vie simple que l'animal possède déjà. L'animal ne propose pas un modèle des vertus inférieures ou un modèle inférieur de vertu, mais une vertu complète que l'homme, à sa façon, peut atteindre, c'est-à-dire, entre autres, en faisant un usage pratique et critique de sa raison. Nous pouvons donc en conclure que le modèle de l'animal dans le cynisme ne s'effectue pas dans le cadre d'une analogie ascendante comme chez Platon ou Plutarque, où l'exemple est situé plus bas que ce par rapport à quoi il est l'exemple, mais d'une analogie descendante, puisque l'animal est moralement supérieur à l'homme n'ayant pas atteint la sagesse. La présence ou l'absence de la raison n'est pas un critère de classement.

Ainsi, si l'exemplarité de l'animal n'est pas exceptionnelle dans l'Antiquité, sa présence dans le cadre cynique est néanmoins tout à fait originale puisqu'elle signifie une supériorité morale réelle et non pas simplement analogique. Mais à quel niveau l'homme doit-t-il imiter l'animal et d'ailleurs, doit-il imiter tous les animaux ? En effet, comme l'a montré 0. Flores-Júnior (2005, p. 135-171), l'animal apparaît également dans le cynisme comme paradigme négatif.

\section{Les limites de l'imitation}

C'est dans une parodie de l'élégie aux muses de Solon, que Cratès, en effet, s'exprime ainsi :
Filles illustres de Mnémosyne et de Zeus Olympien, Muses de Piérie, écoutez ma prière :

Chaque jour donnez à mon ventre sa pâture, à ce ventre qui bien loin de l'esclavage m'assure une vie frugale. $[. .$.

Des avoirs somptueux, je ne veux point en accumuler, en cherchant avidement

Ces avoirs qui font le bonheur de l'escargot et l'aisance de la fourmi !

Non! Je veux participer à la justice et recueillir la richesse

Facile à porter et à se procurer, honorable pour atteindre la vertu.

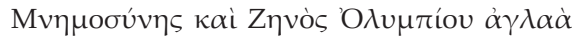
$\tau \varepsilon ́ \kappa v \alpha$,

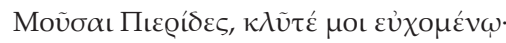

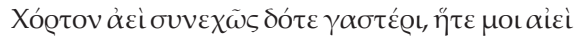

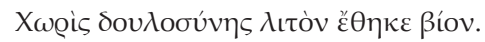

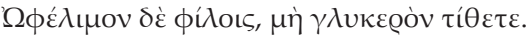

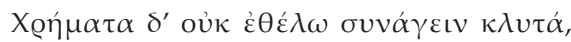

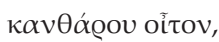

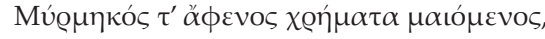

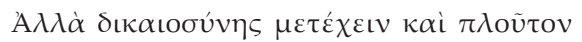
$\dot{\alpha} \gamma \mathrm{\iota} v \varepsilon \tilde{\mathrm{\imath}} \mathrm{v}$

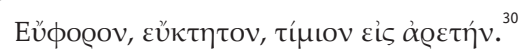

Ainsi, le cynique ne peut pas prendre modèle sur tous les animaux ou tout au moins sur tous leurs comportements. La fourmi, dans la conscience commune, apparaît comme un animal industrieux, s'employant avec obstination à constituer des réserves, à la fois dans les fables d'Ésope, « La cigale et les fourmis » (Ésope, 336) et « La fourmi et l'escarbot » ${ }^{31}$ et chez Aristote ${ }^{32}$. Dans la fable « La fourmi », elle est, de plus, présentée comme une voleuse $^{33}$, et c'est bien cette accumulation de richesses que Cratès condamne. Le statut de l'escarbot est plus difficile à interpréter, puisque chez Ésope, ce dernier ne choisit pas l'accumulation comme la fourmi, ce qui pourrait nous laisser penser qu'il représenterait plutôt la morale cynique. Cependant, par ailleurs, dans la fable « Les deux escarbots »", l'escarbot est représenté comme un être glouton, qui mange une grande quantité de nourriture que, d'autre part, il ne peut pas emporter avec lui, alors
28. Cf. GOULET-CAZÉ, 1993, p. 117-158.

29. Selon la conception cynique de la nature, chaque animal, y compris l'homme, trouve facilement dans son milieu de quoi vivre ; cette autarcie généralisée tend, de fait, à égaliser les conditions, la nature n'étant pas centrée sur l'homme, foyer de l'ordre téléologique providentialiste, mais sur chacun des vivants. A ce niveau là, nous pouvons dire que la notion même de hiérarchie est compromise.

30. Jul., Or. VII, 9, 214b2-214c4 Or. IX [VI], 17, 199d2-200a3 [=SSR VH84], trad. 0. FloresJúnior, art. cit., pp. 143-144, très légèrement modifiée.

31. "Dans la saison d'été, une fourmi rôdant dans la campagne amassait des grains de blé et d'orge, et les mettait en réserve pour s'en nourrir en hiver. Un escarbot l'aperçut et s'étonna de la voir si laborieuse, elle qui travaillait au temps même où les autres animaux, débarrassés de leurs travaux se donnent du bon temps. Sur le moment, la fourmi ne répondit rien; mais plus tard, quand vint l'hiver et que la pluie détrempa les bouses, l'escarbot affamé vint demander à la fourmi l'aumône de quelque aliment. La fourmi lui dit alors; «0 escarbot, si tu avais travaillé au temps où je prenais de la peine et où tu m'injuriais, tu ne manquerais pas à présent de nourriture ». » (Esope, 241, trad. E. Chambry, dans Esope, Fables (1927), Paris, Les Belles Lettres).

32. Cf. Aristote, HA, IX, 38 , 322b19-22, où la fourmi est classée parmi les insectes les plus

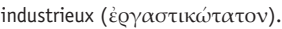
33. « La fourmi d'à présent était autrefois un homme qui, adonné à l'agriculture, ne se contentait pas du produit de ses propres travaux ; il regardait d'un œil d'envie ceux des autres et ne cessait de dérober les fruits de ses voisins. » (Esope, 240, trad. E. Chambry).

34. « Un taureau paissait dans une petite île, et deux escarbots se nourrissaient de sa bouse. À l'arrivée de l'hiver, l'un dit à l'autre qu'il voulait passer sur le continent, afin que, étant seul, son camarade eût de la nourriture en suffisance, tandis que lui s'en irait là-bas pour y passer l'hiver. Il ajouta que, s'il y trouvait de la pâture en abondance, il lui en apporterait. Or, arrivé sur le continent, il y rencontra des bouses nombreuses et fraîches; il s'y établit et s'en nourrit. L'hiver passé, il revint dans l'île. Son 
camarade le voyant gras et en bon corps, lui rappela sa promesse et lui reprocha de ne lui avoir rien rapporté. «Ne t'en prends pas à moi, répondit-il, mais à la nature du lieu : il est possible d'y trouver à vivre, mais impossible d'en emporter quoi que ce soit». On pourrait appliquer cette fable ceux qui poussent l'amitié jusqu'à régaler leurs amis, mais pas plus loin, et qui refusent de leur rendre aucun service. » (Esope, 149, trad. E. Chambry)

35. L'autre escarbot célèbre est grand bousier qui apparait dans le prologue de La paix d'Aristophane et que deux serviteurs doivent nourrir de galettes d'excréments. Pour une étude de la conceptio biologique et symbolique de l'escarbot dans l'Antiquité, cf. NICOLAS-DUVAL, 2009, p. 107 que Cratès cherche une richesse « facile à porter »

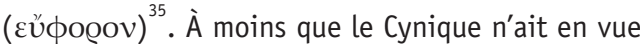
la grande boulette d'excréments que le bousier roule péniblement, de laquelle il est censé se nourrir, et dans laquelle pour Aristote (HA, V,19, 552a17-19) il hiberne et se reproduit. Quoi qu'il en soit, il apparaît que tous les animaux, ou du moins tous leurs comportements, ne sont pas à imiter.

Est-ce parce que la vie n'est pas facile pour tous les animaux, alors qu'elle l'est pour l'homme, de sorte qu'il ne faut prendre pour modèle que ceux qui vivent au jour le jour, qui ne sont pas industrieux ? Mais, en ce cas, cela signifie-t-il que tous les animaux ne sont pas exempts de souci et donc qu'ils ne sont pas tous heureux, de sorte qu'il faudrait établir une hiérarchie morale au sein du monde animal ? Dans un contexte cynique, cela semble difficile à soutenir : la fourmi et l'escarbot, malgré leurs comportements, n'en sont pas moins heureux, puisqu'ils ne sont victimes ni des illusions humaines, ni de la crainte de la mort. Ce texte montre simplement que, s'il faut regarder vers l'animal pour y trouver le bonheur que réserve la nature, il ne faut pas imiter en tout point ses comportements, lesquels sont d'ailleurs contradictoires. Si l'animal est un modèle, il peut aussi être imité de façon perverse par les insensés qui y cherchent une justification de leurs erreurs. En somme, seul le sage connaît, en toute certitude, pour en avoir fait lui-même l'épreuve, ce qui en l'animal est à imiter.

Nous pouvons même retrouver cette ambivalence de la référence à l'animal, dans ces deux apophtegmes qui visent à montrer la transformation par Diogène du stigmate lié au surnom insultant «Chien » en revendication positive.

Alors que Polyxène le dialecticien s'indignait parce que certains l'interpellaient par le mot " chien ", il dit : “ Toi aussi appelle-moi " chien ". En effet, Diogène n'est pour moi qu'un surnom ; c'est un chien que je suis, mais de ces chiens de bonne race (gennaiôn) qui montent la garde sur leurs amis. (Gnom. Vat. 743, n. $194[=$ VB 149])

Diogène choisit donc de s'identifier de façon paradoxale à l'animal, mais, au sein même de la race canine, il sait précisément à quel type de chiens il faut s'identifier. Il en vient même parfois à renverser les comportements des chiens empiriques.

Diogène disait : "les autres chiens mordent leurs ennemis, mais moi je mords mes amis afin de les sauver".(Stobée III 13, 44 [= VB 149])

Il mord métaphoriquement ses amis pour les protéger de leurs propres illusions, et n'apprécie donc guère la douceur proverbiale du chien à l'égard des familiers combinée à son agressivité à l'égard des étrangers (les ennemis) qui, pour Platon, était essentielle (Rsp., 375c-e).

Ne faut-il pas voir là toutefois une forme de circularité, l'animal était censé nous montrer ce qu'est la vie heureuse, mais à condition que nous sachions correctement l'interpréter, c'est-à-dire à condition que nous sachions déjà en quoi elle consiste ? Seule est concevable l'imitation d'un modèle, non pas donné, mais construit à l'image de la norme qu'on se donne. L'exemplum, contrairement à ce qu'il promet, ne manifeste pas immédiatement la vertu, il ne fait qu'illustrer la précompréhension que nous en avons. Cette circularité est particulièrement indiscutable dans le cas d'un exemple animal, cependant cette situation n'est pas exceptionnelle puisqu'elle existe aussi lorsque le modèle est humain. Ainsi Socrate, modèle du philosophe pour Platon, met-il en vers une fable d'Ésope avant de mourir ${ }^{36}$, et pourtant versifier Ésope n'est pas devenu, pour son élève, un précepte philosophique intangible : imiter un modèle moral ne revient jamais à en reproduire tous les comportements, mais à en retrouver la disposition intérieure.

Ce qui fait l'originalité du cynisme n'est donc pas d'établir des analogies entre l'homme et l'animal, voire même de constituer l'animal en modèle, mais de renverser les cadres généraux de l'entreprise analogique, d'user de la raison, non pas pour dépasser l'animal, mais pour retrouver ce qui en lui se rapproche de l'autarcie divine.

\section{BIBLIOGRAPHIE}

BILLAULT, A. (2005). Le modèle animal dans le traité de

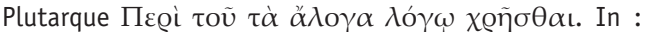
J. Boulogne (éd.). Les Grecs de l'Antiquité et les animaux. 
Le cas remarquable de Plutarque. Villeneuve d'Ascq, Université Charles-de-Gaulle Lille 3, p. 33-42.

BRÉCHET, C. (2005). La philosophie de Gryllos. In : J. Boulogne (éd.). Les Grecs de l'Antiquité et les animaux. Le cas remarquable de Plutarque. Villeneuve d'Ascq, Université Charles-de-Gaulle Lille 3, p. 43-61.

COPPENS, Y., PICQ, P. (dir.) (2001). Aux Origines de l'humanité, Le propre de l'homme, Paris, Fayard, tome 2.

DESCOLA, P. (2005). Par-delà nature et culture. Paris, Gallimard.

FLORES-JÚNIOR, 0. (2005). Cratès, la fourmi et l'escarbot, Philosophie antique, n. 5, p. 135-171.

GOLDSCHMIDT, V. (1985). Le paradigme dans la dialectique platonicienne. Paris, Vrin.

GONTIER, Th. (1999). L'homme et l'animal. La philosophie antique. Paris, PUF (coll. «Philosophies »).
GOULET-CAZÉ, M.-0. (1993). Les premiers Cyniques et la religion. In : M.-0. Goulet-Cazé, R. Goulet (éds). Le cynisme ancien et ses prolongements. Actes du colloque international du C.N.R.S. (Paris 22-25 juillet 1991). Paris, PUF, p. 117-158.

GOULET-CAZÉ, M.-0. (2003). Les Kynika du stoïcisme. Stuttgart, Franz Steiner Verlag.

HUSSON, S. (2011). La République de Diogène. Une cité en quête de la nature. Paris, Vrin.

NICOLAS-DUVAL, B. (2009). L'escarbot, un topos de l'osmologie grecque, Schedae, Prépublication n. 7, Fasc. 1, p. 107-123, consulté le 30/11/12 sur le site de l'Université de Caen : www.unicaen.fr/puc/ecrire/ preprints/preprint0072009.pdf.

RIGOLOT, F. (1998). The Renaissance Crisis of Exemplarity, The Journal of the History of ldeas, n. 59, v. 4, p. 557-563.

Recebido em setembro de 2012 e aprovado em setembro de 2012. 